\title{
Design Approach of Grid Coupled Solar Inverter
}

\author{
Bandla Pavan $\mathrm{Babu}^{1,}$ V.Indragandhi ${ }^{2}$, R. RajaSingh ${ }^{3}$ and Ramani Kannan ${ }^{4}$ \\ ${ }^{1}$ Vellore Institute of Technology, Vellore \\ ${ }^{2,3}$ Vellore Institute of Technology, Vellore \\ ${ }^{4}$ Department of Electrical and Electronic Engineering, Universiti Teknologi PETRONAS, Malaysia
}

\begin{abstract}
In novel times, more demands are gained by grid connected solar inverters along with the upgrading of the solar energy generation. In grid connected solar power generation, if there is increase in placing more number of solar inverters it may result in effecting the power quality issues. Solar Grid Tied Inverter system is a electrical power generating system that is coupled to the functioning power grid. This power generating system unit consists of elements like Photovoltaic array, DC to DC converter, DC to AC converter, single phase/three phase converter, and AC Source. The inverter converts DC power generated by the array of photovoltaic cells into $\mathrm{AC}$ power and this generated $\mathrm{AC}$ power is fed to the connected AC loads. If the amount power generation is exceeding the power demanded, excess AC power is supplied to the grid. With this novel approach we can minimize the use of the AC power from the power grid and also even make a generation to the power grid and this is measured with help of net metering. In this paper designing of $281.6 \mathrm{Kw}$ grid tied solar power generation and technical specifications required for them are proposed.
\end{abstract}

\section{Introduction}

Indian government has a quest to install hundred Giga Watts of solar power energy by the end of 2022[1-3].Due to the increase in energy demands for the last few decades; there is increase in natural problems and reduction of fossil fuel assets. This leads to think about for another sources i.e., renewable energy sources especially Solar Energy. With thereduction in solar cells cost, it is economically feasible for solar energy conversion to electric energy [47].The solar electric power generation system includes PV panels, DC to DC converter, DC to AC inverter, DC cabling.Maximum power point tracking (MPPT) is a technique preferred to increase the overall efficiency of photovoltaic systems by drawing maximum power available at all times irrespective of irradiation and temperature conditions. Without MPPT technique when the power generated by PV systems connected directly it results in poor efficiency of the system. Grid tied inverter is different from a traditional inverter which is available. The following requirements are a must and need to be fulfilled before getting connected with the grid[8-11]. Inverter output voltage magnitude must be same as functioning grid voltage, phase and phase sequence of inverter output must be same as functioning grid phase and phase sequence, the inverter output frequency must be same as functioning grid frequency which is shown in Fig 1 shown below.

\footnotetext{
${ }^{1}$ Corresponding author : author@email.org
} 


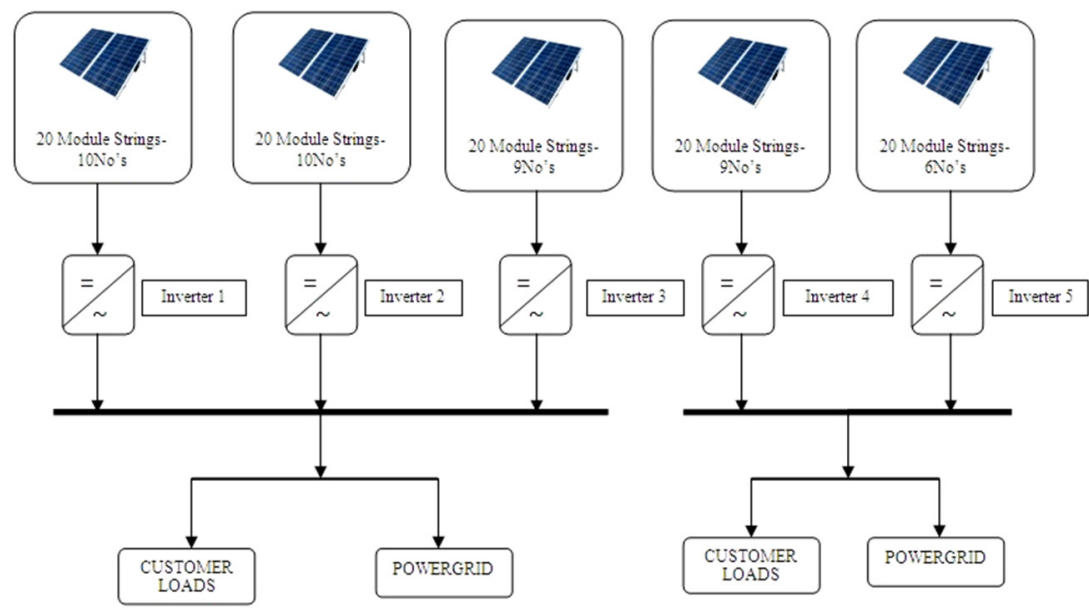

Fig.1. Proposed approach for Designing PV Solar Plant

\section{Design considérations}

\subsection{PV Panel}

To test the performance of Solar PV cells, there are various standards testing conditions of PV segments. Majority of the test conditions includes Standard Test Conditions (STC) as shown in Table 2, Normal Operating Cell Temperature (NOCT) as shown in Table 3 Low Irradiance Conditions (LIC), High Temperature Conditions (HTC)and Low Temperature Conditions (LTC). The International Electrotechnical Commission (IEC) publishedthe IEC 61853 standard "Photovoltaic Module. Power Rating" which requires testing based on a variety of climatic and geographic conditions and includes HTC, LIC, HTC, NOCT and STC.[12-13]

Table 1. PV Panel Specifications

\begin{tabular}{|l|l|}
\hline cell & Poly $156.75^{*} 156.75 \mathrm{~mm}$ \\
\hline weight & $222.5 \mathrm{~kg} \pm 3 \%$ \\
\hline Dimensions & $1960^{*} 991^{*} 40 \mathrm{~mm}$ \\
\hline Cable cross section size & $4 \mathrm{~mm}^{2}$ \\
\hline No of cells & $72\left(6^{*} 12\right)$ \\
\hline Junction Box & IP67,3 diodes \\
\hline connector & MC4 Compatible \\
\hline Packaging configuration & 27 per pallet \\
\hline
\end{tabular}

Table 2. Electrical Parameters at STC 


\begin{tabular}{|c|c|c|c|c|c|}
\hline TYPE & $\begin{array}{c}\text { JAP72S01 } \\
-310 / \mathrm{SC}\end{array}$ & $\begin{array}{c}\text { JAP72S01 } \\
-315 / \mathrm{SC}\end{array}$ & $\begin{array}{c}\text { JAP72S01 } \\
-320 / \mathrm{SC}\end{array}$ & $\begin{array}{c}\text { JAP72S01 } \\
-325 / \mathrm{SC}\end{array}$ & $\begin{array}{c}\text { JAP72S01 } \\
-330 / \mathrm{SC}\end{array}$ \\
\hline $\begin{array}{l}\text { Rated Maximum } \\
\text { power }\left(\mathrm{P}_{\max }\right)[\text { Watts }]\end{array}$ & 310 & 315 & 320 & 325 & 330 \\
\hline $\begin{array}{l}\text { Open circuit voltage } \\
\text { (Voc)[Volts] }\end{array}$ & 45.56 & 45.85 & 46.12 & 46.78 & 46.40 \\
\hline $\begin{array}{c}\text { Maximum power } \\
\text { voltage }\left(V_{m p}\right)[\text { Volts }]\end{array}$ & 36.89 & 37.09 & 37.28 & 37.39 & 37.65 \\
\hline $\begin{array}{l}\text { Short circuit current (Isc) } \\
\text { [Amps] }\end{array}$ & 8.92 & 9.01 & 9.09 & 9.17 & 9.28 \\
\hline $\begin{array}{l}\text { Maximum power current } \\
\text { (IMP) [Amps] }\end{array}$ & 8.40 & 8.49 & 8.69 & 8.69 & 8.77 \\
\hline Module Efficiency [\%] & 15.96 & 16.22 & 16.73 & 16.47 & 16.99 \\
\hline Power Tolerance & & & $0 \sim+5 \mathrm{~W}$ & & \\
\hline $\begin{array}{l}\text { Temperature Coefficient } \\
\text { of Isc }\left(\alpha \_I_{S C}\right)\end{array}$ & & & $+0.058 \% /{ }^{\circ} \mathrm{C}$ & & \\
\hline $\begin{array}{l}\text { Temperature Coefficient } \\
\text { of }\left(\beta \mathrm{V}_{o c}\right)\end{array}$ & & & $-0.330 \% /{ }^{\circ} \mathrm{C}$ & & \\
\hline $\begin{array}{l}\text { Temperature Coefficient } \\
\text { of } \operatorname{Pmax}\left(\gamma_{-} P_{m p}\right)\end{array}$ & & & $-0.410 \% /{ }^{\circ} \mathrm{C}$ & & \\
\hline $\begin{array}{l}\text { Standard Test Condition } \\
\text { (STC) }\end{array}$ & \multicolumn{5}{|c|}{ Irradiance $1000 \mathrm{w} / \mathrm{m}^{2}$, cell temperature $25^{\circ} \mathrm{c} \mathrm{AM} 1,5 \mathrm{G}$} \\
\hline
\end{tabular}

Table 3. Electrical Parameters at NOCT

\begin{tabular}{|c|c|c|c|c|c|}
\hline TYPE & $\begin{array}{l}\text { JAP72S01- } \\
\text { 310/SC }\end{array}$ & $\begin{array}{l}\text { JAP72S01- } \\
315 / \text { SC }\end{array}$ & $\begin{array}{l}\text { JAP72S01- } \\
320 / \text { SC }\end{array}$ & $\begin{array}{l}\text { JAP72SC01- } \\
\text { 325/SC }\end{array}$ & $\begin{array}{l}\text { JAP72SC01- } \\
\text { 330/SC }\end{array}$ \\
\hline Max Power $\left(\mathrm{P}_{\max }\right)[$ Watts $]$ & 229 & 233 & 237 & 241 & 244 \\
\hline $\begin{array}{l}\text { Open circuit voltage }\left(\mathrm{V}_{o c}\right) \\
{[\text { Volts }]}\end{array}$ & 42.63 & 42.84 & 43.04 & 43.24 & 43.41 \\
\hline $\begin{array}{l}\text { Max Power voltage } \\
\left(\mathrm{V}_{\mathrm{mp}}\right)[\text { Volts }]\end{array}$ & 34.32 & 34.45 & 34.64 & 34.82 & 35.03 \\
\hline $\begin{array}{l}\text { Short circuit current } \\
\left(\mathrm{I}_{\mathrm{s} C}\right)[\mathrm{Amps}]\end{array}$ & 7.18 & 7.35 & 7.29 & 7.35 & 7.40 \\
\hline $\begin{array}{l}\text { Max power current }\left(\mathrm{I}_{\mathbb{M P}}\right) \\
{[\mathrm{Amps}]}\end{array}$ & 6.68 & 6.91 & 6.84 & 6.91 & 6.97 \\
\hline $\begin{array}{l}\text { Normal } \\
\text { Operating Cell Temperature } \\
\text { (NOCT) }\end{array}$ & \multicolumn{5}{|c|}{ Irradiance $800 \mathrm{w} / \mathrm{m}^{2}$, ambient temperature $20^{\circ} \mathrm{c}$, Wind Speed $1 \mathrm{~m} / \mathrm{S}, \mathrm{Am}, 1.5 \mathrm{~g}$} \\
\hline
\end{tabular}

Table 4. operating conditions

\begin{tabular}{|l|l|}
\hline Maximum system voltage & $1000 \mathrm{vDC}($ IEC) \\
\hline Operating temperature & $-40^{\circ} \mathrm{C} \sim+85^{\circ} \mathrm{C}$ \\
\hline Maximum series fuse & $20 \mathrm{~A}$ \\
\hline Maximum Static Load, Front & $5400 \mathrm{pa}$ \\
Maximum Static Load, Back & $2400 \mathrm{pa}$ \\
\hline NOCT & $45 \pm 2^{\circ} \mathrm{C}$ \\
\hline Application class & Class A \\
\hline
\end{tabular}

\subsection{PV Panel Characteristics}


When designing a PV system, four factors namely operating temperature, solar intensity, sun's incident angle and I-V (Fig 2) \& P-V (Fig 3) performance characteristics are considered.

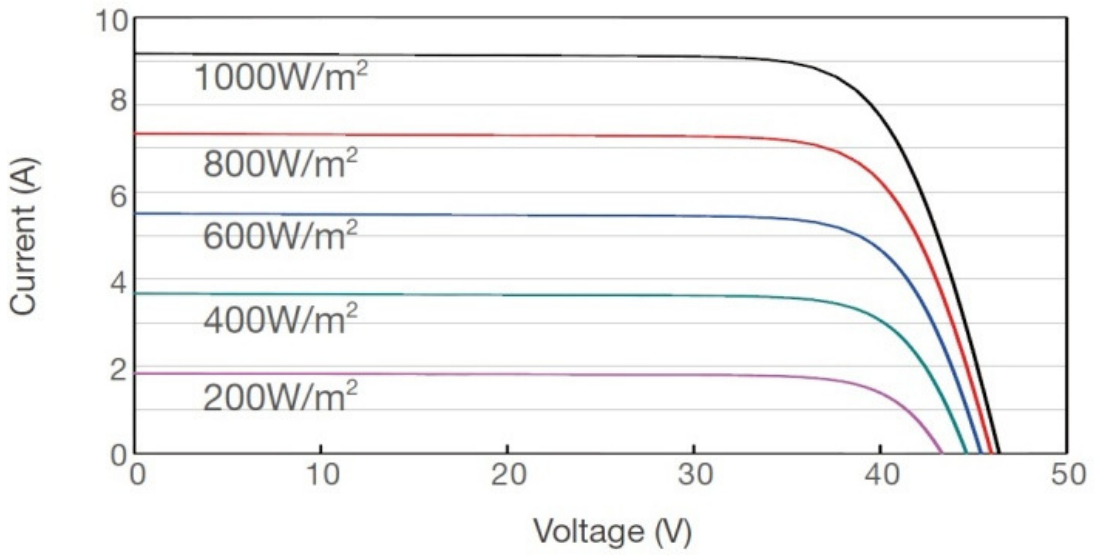

Fig. 2.Current - Voltage Curve (JAP72S01-325/SC)[13-14]

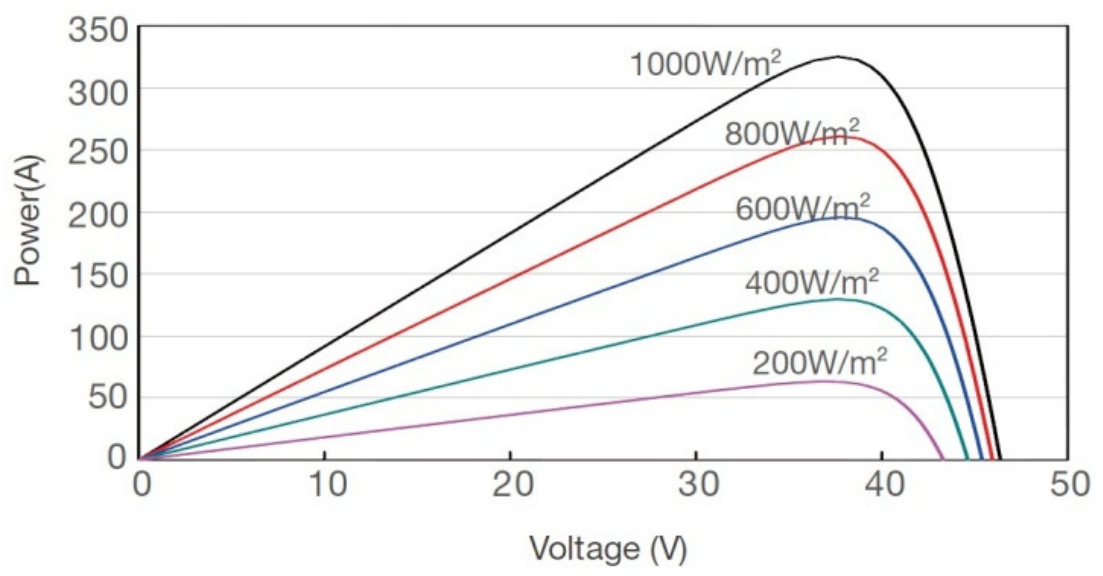

Fig. 3. Power - Voltage Curve (JAP72S01-325/SC) [13-14]

This graphical representation Fig 2 \& Fig 3 shows the performance characteristics which is of unique to each and every model of solar panel and this graph is termed as the "I-V" curve(Fig 2) and "P-V" curve(Fig 3) which gives the relationshipbetween current (I)voltage $(\mathrm{V})$ and Power $(\mathrm{P})$ - Voltage $(\mathrm{V})$ under prevailingconditions of temperature and sunlight. There exists mutiple number of I-V \& P-V curves for one particular module which is shown in Fig 2 \& Fig 3 is due to the different operating conditions that would affect the STC rating parameters such as the temperature, air mass, irradiance, etc., and that's a lot of possible graphs.[12-13]

\subsection{PV Inverter}

"RPI-M50A"transformer less Photovoltaic inverter is the glossy, least possible and first wall mutable $50 \mathrm{Kw}$ string inverter in the present world. With such compressed size and less 
weight, RPI-M50A tenders high design tractability for different capacities of Photovoltaic plants. Higher level of protection and enhanced durability in a severe outdoor environment provided by IP65 enclosure. The DC Specifications and obtained AC parameters are given in Table 5 \& Table 6 respectively [13-16].

Table 5. DC Input to the Inverter

\begin{tabular}{|c|c|}
\hline Input (DC) & RPI-M50A \\
\hline Recommended max. Dc power & $63 \mathrm{kWP}$ \\
\hline Max. Input voltage & $1000 \mathrm{~V}$ \\
\hline Dc voltage range & $200-1000 \mathrm{~V}$ \\
\hline Start-up voltage & $>250 \mathrm{~V}$ \\
\hline Start-up power & $40 \mathrm{~W}$ \\
\hline MPPT voltage range & $200-1000 \mathrm{~V}$ \\
\hline MPP voltage range, full power & $520-800$ \\
\hline Nominal dc voltage & $600 \mathrm{~V}$ \\
\hline Max. Input current per MPPT & $50 \mathrm{~A}$ \\
\hline Total input current & $100 \mathrm{~A}$ \\
\hline Maximum short circuit current & $60 \mathrm{~A} / 60 \mathrm{~A}$ \\
\hline No. Of independent MPP trackers & 2 \\
\hline Unbalanced input (\%) & $33 / 67$ \\
\hline Input connection type & 12 pair MC4 \\
\hline Dc disconnection switch & Yes (inbuilt) \\
\hline
\end{tabular}

Table 6. AC Output of the Inverter

\begin{tabular}{|c|c|}
\hline Rated output power & $50 \mathrm{KVA}$ \\
\hline Maximum output power & $55 \mathrm{KVA}$ \\
\hline Rated output current & $76 \mathrm{~A}$ \\
\hline Max. output current & $80 \mathrm{~A}$ \\
\hline Inrush current & $200 \mathrm{~A} / 100 \mu \mathrm{s}$ \\
\hline Nominal AC voltage & $3 \mathrm{Ph}, 400 \mathrm{~V}$ \\
\hline AC voltage range & $\begin{array}{c}400 \mathrm{~V} \pm 20 \% \\
(320 \sim 480)\end{array}$ \\
\hline Nominal frequency & $50 \mathrm{HZ}$ \\
\hline Frequency range & $45 \mathrm{HZ}-55 \mathrm{HZ}$ \\
\hline $\begin{array}{c}\text { Power factor at rated } \\
\text { power }\end{array}$ & $\mathrm{UNITY}$ \\
\hline $\begin{array}{c}\text { Reactive power } \\
\text { (Adjustable) }\end{array}$ & $\begin{array}{c}0.8 \mathrm{Lagging} \sim 0.8 \\
\text { leading }\end{array}$ \\
\hline $\begin{array}{c}\text { Harmonics } \\
\text { Parmed }\end{array}$ \\
\hline $\begin{array}{c}\text { DC injection } \\
\text { Power }\end{array}$ \\
\hline $\begin{array}{c}\text { No. of conductors } \\
\text { (user settable) }\end{array}$ & $\begin{array}{c}<0.5 \% \text { at Rated } \\
\text { Output Current }\end{array}$ \\
\hline
\end{tabular}




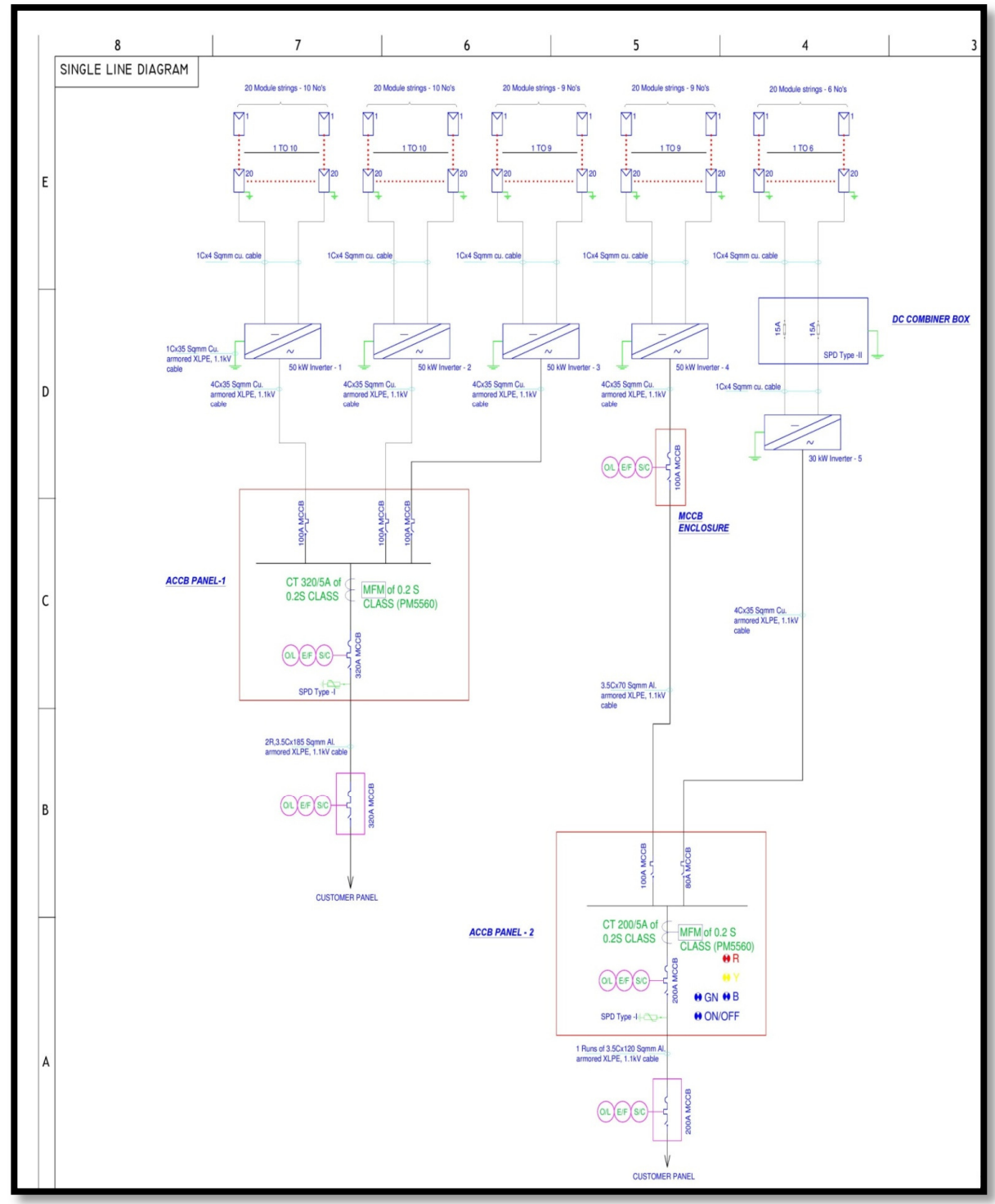

Fig. 4. Single Line Diagram of grid tied 281.6KW Solar Power Generation with inverter modules. 


\section{Conclusion}

In this, a photovoltaic system for interconnection to the functioning electrical power grid is designed. Validity of the designed Photovoltaic system with the grid coupled can be verified through the experimental results. The relative cost of this solar inverter will also be less as there is minimal number of power devices and dual MPPT is used to execute this composition. It will be also clear from the result, that the obtained output of the inverter modules at different solar panel voltages levels can be prolonged within the particular regulation limits of the functioning electrical power grid. Grid tied photovoltaic systems, with all the above benefits made the inverter composition highly feasible.

\section{References}

1. P. Patel, K. R. Sekhar and Y. Patel, "A novel control strategy to enhance the current quality in grid tied solar inverter," 2016 IEEE International Conference on Power Electronics, Drives and Energy Systems (PEDES), Trivandrum, 2016, pp.1-6.

2. A. Salazar-Llinas, A. Ginart and C. Restrepo, "Observer Based Sensor Fault Tolerant for Grid Tied - Solar Inverters," 2014 Sixth Annual IEEE Green Technologies Conference, Corpus Christi, TX, 2014, pp.69-74.

3. Y. Wang and F. Wang, "Novel Three-Phase Three-Level-Stacked Neutral Point Clamped Grid-Tied Solar Inverter With a Split Phase Controller," in IEEE Transactions on Power Electronics, vol. 28, no. 6, pp. 2856-2866, June 2013.

4. M. NahidHossain, TusharKantiRouth, Abdul Hamid Bin Yousuf, M. M. Asasduzzaman, M. IqbalHossain and U. Husnaeen, "Design and development of a grid tied solar inverter," 2012 International Conference on Informatics, Electronics \& Vision (ICIEV), Dhaka, 2012, pp. 1054-1058.

5. B. G. Devi and M. Mahesh, "A brief survey on different multilevel inverter topologies for grid-tied solar photo voltaic system," 2017 IEEE International Conference on Smart Energy Grid Engineering (SEGE), Oshawa, ON, 2017, pp. 51-55.

6. J. L. M. Yi, R. T. Naayagi and T. Logenthiran, "Modelling and implementation of single phase dual stage grid-tied solar power inverter," 2016 IEEE Region 10 Conference (TENCON), Singapore, 2016, pp. 1879-1883.

7. D. Hameed, S. Hamayoon, A. A. Malik and O. A. Ansari, "Solar grid-tied inverter, with battery back-up, for efficient solar energy harvesting," 2016 IEEE Smart Energy Grid Engineering (SEGE), Oshawa, ON, 2016, pp. 95-99.

8. Y. Wang and F. Wang, "Novel Three-Phase Three-Level-Stacked Neutral Point Clamped Grid-Tied Solar Inverter With a Split Phase Controller," in IEEE Transactions on Power Electronics, vol. 28, no. 6, pp. 2856-2866, June 2013.

9. W. Liu, K. Wang, H. S. h. Chung and S. T. h. Chuang, "Modeling and Design of Series Voltage Compensator for Reduction of DC-Link Capacitance in Grid-Tie Solar Inverter," in IEEE Transactions on Power Electronics, vol. 30, no. 5, pp. 2534-2548, May 2015.

10. P. H. Kydd, J. R. Anstrom, P. D. Heitmann, K. J. Komara and M. E. Crouse, "VehicleSolar-Grid Integration: Concept and Construction," in IEEE Power and Energy Technology Systems Journal, vol. 3, no. 3, pp. 81-88, Sept. 2016.

11. M. K. Das, K. C. Jana and A. Sinha, "Performance evaluation of an asymmetrical reduced switched multi-level inverter for a grid-connected PV system," in IET Renewable Power Generation, vol. 12, no. 2, pp. 252-263, 252018. 
12. Website: www.en.jasolar.com

13. Website:www.deltaelectronicsindia.com

14. Indragandhi, V \& V, Subramaniyaswamy\& R, Logesh. (2017). Resources, configurations, and soft computing techniques for power management and control of PV/wind hybrid system. Renewable and Sustainable Energy Reviews. 69. 129-143. 10.1016/j.rser.2016.11.209.

15. Veena, P. \&Indragandhi, V. \&Jeyabharath, R. \&Subramaniyaswamy, V., 2014. "Review of grid integration schemes for renewable power generation system," Renewable and Sustainable Energy Reviews, Elsevier, vol. 34(C), pages 628-641.

16. T. Arunkumari, V. Indragandhi, An overview of high voltage conversion ratio DC-DC converter configurations used in DC micro-grid architectures, In Renewable and Sustainable Energy Reviews, Volume 77, 2017, Pages 670-687, ISSN 1364-0321. 